2018-08

\title{
Intention insertion: Activating an action's perceptual consequences is sufficient to induce non-willed motor behavior
}

\section{Colton, J}

http://hdl.handle.net/10026.1/11293

\section{$10.1037 / x g e 0000435$ \\ Journal of Experimental Psychology: General \\ American Psychological Association}

All content in PEARL is protected by copyright law. Author manuscripts are made available in accordance with publisher policies. Please cite only the published version using the details provided on the item record or document. In the absence of an open licence (e.g. Creative Commons), permissions for further reuse of content should be sought from the publisher or author. 
Running head: INTENTION INSERTION

Intention insertion: activating an action's perceptual consequences is sufficient to induce non-willed motor behaviour.

James Colton, Patric Bach, Ben Whalley \& Christopher Mitchell

School of Psychology, University of Plymouth, Devon, UK

Running head: INTENTION INSERTION

Word count: 4,743 words, 2 figures.

\section{Correspondence:}

James Colton

School of Psychology

University of Plymouth

Drake Circus, Devon PL4 8AA, UK

james.colton@plymouth.ac.uk 


\title{
1INTENTION INSERTION
}

\begin{abstract}
It feels intuitive that our actions are intentional, but there is considerable debate about whether (and how) humans control their motor behaviour. Recent ideomotor theories of action argue that action intentions are fundamentally perceptual, that actions are not only controlled by anticipating - imagining - their intended perceptual consequences, but also initiated when this action effect activation is strong. Here, we report a study (plus a replication) that provides direct evidence for this proposal, showing that even non-intended actions are executed when their effects are activated strongly enough. Participants mentally rehearsed a movement sequence and were unexpectedly presented with salient visual cues that were either compatible or incompatible with their currently imagined action. As predicted by ideomotor theories, the combined activation through imagery and perception was sufficient to trigger involuntary actions, even when participants were forewarned and asked to withhold them. Ideomotor cues, therefore, do not only influence pre-planned responses but can effectively insert intentions to act, creating behaviour de novo, as predicted from ideomotor theories of action control.
\end{abstract}

Keywords: Intentionality; action initiation; mental imagery; action planning; motor control 


\section{INTENTION INSERTION}

\section{Intention insertion: activating an action's perceptual consequences is sufficient to induce non-willed motor behaviour.}

Willed action is one of the biggest puzzles in psychology: do humans will their actions, or do they just follow previously established stimulus-response associations, or respond to affordances offered by the environment (Tucker \& Ellis, 1998)? While a long tradition of psychological research, starting with behaviourism, has denied the possibility of voluntary action or at least exempted it from psychological enquiry, a parallel tradition has made it its central topic. Ideomotor theories argue that people do have voluntary control over their actions, and that these action intentions have a fundamentally perceptual format (Hommel, 2009; Hommel, Müsseler, Aschersleben, \& Prinz, 2001; James, 1890; Ondobaka \& Bekkering, 2012; Pacherie \& Haggard, 2010; Prinz, 1997; Shin, Proctor, \& Capaldi, 2010). To initiate an action, these models argue, humans simply bring to mind - imagine - the desired outcome: how the action would look, feel and sound, and how it would affect the environment. Automatic motor processes make this imagined action reality. Ideomotor models - and more recently those based on predictive processing (e.g. Adams, Shipp, \& Friston, 2013; Friston, 2011) - argue that perceptual control of action is possible because people, through a lifetime of interactions, form associations between their motor behaviours and the effects that these behaviours reliably produce, for example, that tensing a specific muscle group will pull one's hand to the left, or that pressing a brake pedal will slow a car (Turella et al., 2016). As these associations are formed, willed behaviour becomes possible (James, 1890): one merely needs to think of (i.e. imagine) the consequences one wants to achieve, and via the now established associations the relevant motor behaviours are elicited. After sufficient practice, these acts of motor imagery become so effective that they occur largely outside of awareness: we imagine our hand moving, and it does. 


\section{INTENTION INSERTION}

Strikingly, research has largely ignored this intentional or initiating role of action effect activation, focussing only on their contribution to selecting or shaping our actions. For example, it has been shown that participants execute an action more quickly (and make fewer errors) when primed, just prior to execution, with an image of the consequences of the intended act, be they "proximal" consequences on the actor's own body (e.g. a hand posture or movement of a body part; see Bach, Peatfield, \& Tipper, 2007; Hommel, 1995; Brass, Bekkering, \& Prinz, 2001) or "distal" consequences in the environment (e.g. a light switching on, a tone playing; Elsner \& Hommel, 2001). This facilitation even happens when the action's effects are not perceived, but merely anticipated (because they reliably follow the action in the response-effect compatibility paradigm, e.g. Kunde, Koch, \& Hoffmann, 2004; see Badets, Koch, \& Philipp, 2016 for review) and neuroimaging studies have shown that action planning involves activation in lower-level perceptual regions that represent the specific outcome one wants to produce (Kühn, Keizer, Rombouts, \& Hommel, 2011; van Steenbergen et al., 2017; Zimmermann, Mars, Lange, Toni, \& Verhagen, 2018; Zimmermann, Verhagen, de Lange, \& Toni, 2016). Yet, while such studies provide converging evidence that ideomotor cues contribute to the selection of an action against alternatives or shape its kinematics (Bach, Griffiths, Weigelt, \& Tipper, 2010) they do not test the central assumption of ideomotor models: that activation of an action's perceptual effect is tantamount to forming an intention to act, in short, that activation of an action's effects does not only determine which action is selected or how it is executed, but whether it is executed at all.

Recent research conceptualises action initiation in terms of an integration-to-bound mechanism, where evidence for an array of appropriate actions accumulates until a threshold is reached, and the most suitable action is executed, triggering a cascade of downstream motor processes (Churchland, Kiani, \& Shadlen, 2008; Murakami, Vicente, Costa, \& Mainen, 


\section{INTENTION INSERTION}

2014; Schurger, Mylopoulos, \& Rosenthal, 2016). If the assumptions of ideomotor models are taken seriously, then the evidence accumulated by such mechanisms is nothing else than the activation of multimodal action effect codes: how strongly the perceptual consequences of the action are mentally represented (see Friston, 2011, for similar arguments in predictive processing frameworks). An imagined action can become overt behaviour if the anticipated action effects become sufficiently active.

Tentative support for the equivalence of action effect anticipation and intention comes, on the one hand, from the observation that motor imagery increases corticospinal excitability to the imagined effector (Vargas et al., 2004) and research on $19^{\text {th }}$ century parlour games such as Chevreul's magic swinging pendulum or the Ouija board. In these games, participants experience movements they did not consciously intend to produce (and therefore attribute to supernatural causes), but which nevertheless reflect their perceptual expectations of what will happen (Chevreul, 1833; Easton \& Shor, 1975, 1976, 1977). However, such effects are typically small and fall far below the threshold for fully formed responses, even if amplified either by these "magic" devices or transcranial magnetic stimulation. Others have therefore argued against such an initiating function of action effect anticipation, providing neuroimaging and behavioural evidence, for example, that motor imagery mainly affects the planning of the action, specifying its form after initiation (Bach, Allami, Tucker, \& Ellis, 2014; Caldara et al., 2004) and that different processes energise and release the action (Tecuapetla, Jin, Lima, \& Costa, 2016). Indeed, it has been argued that the effects of ideomotor cues on action execution reflect interference rather than facilitation effects (Ramsey, Cumming, Eastough, \& Edwards, 2010) and would therefore - per definition only be able to prevent, not cause, action. 


\section{INTENTION INSERTION}

This study tested for the first time whether the activation of an action's effects is sufficient to cause participants to execute full actions, even if none were previously intended (and had to be actively withheld). It relies on the assumption of ideomotor models that perception, action, and imagery operate on the same or commensurable codes and can therefore summate (Berends, Wolkorte, Ijzerman, \& Van Putten, 2013; Hommel et al., 2001; Kunde et al., 2004; Prinz, 1997; Vogt, Di Rienzo, Collet, Collins, \& Guillot, 2013; Waszak, Cardoso-Leite, \& Hughes, 2012). We therefore asked participants, in each trial, to imagine sequences of left and right index finger presses. In some trials, we presented them with visual stimuli, which could either match the currently imagined action in the sequence (e.g. seeing the same finger depressed) or mismatch with it (e.g. a different finger being depressed). Participants were instructed to ignore these images and withhold any responses, but the effect codes activated by perception and imagery should nevertheless summate (see Kunde et al., 2004) and (sometimes) trigger involuntary action, when their activation becomes super-threshold. Crucially, manipulating the congruence of imagined and observed actions allows us to dissociate involuntary movements that result from the premature release of pre-planned actions due to a startle response (Brown et al., 1991; Maslovat, Chua, \& Hodges, 2013) from those that genuinely result from the summation of action effect codes. In the latter case, involuntary responses should be more frequent when the imagined action and cued action effects are congruent (such that activation can sum up) than when they are incongruent (when such an addition is not possible). Further, these action slips should primarily reflect the imagined action and not the alternative response, at least as long as imagined and observed actions are congruent.

\section{Method}

\section{Participants}




\section{INTENTION INSERTION}

32 participants took part in the experiment (21 female; 5 Left-handed; Age in years: $M=22.1$, $S D=3.5$ ), recruited from the Plymouth University’s paid participation pool (remunerated $£ 4$ ). The study was approved by Plymouth University's ethics committee, in according with the declaration of Helsinki. The sample size was based on a series of pilot studies in which we established the effect and then replicated it here, using identical participant numbers, analyses and exclusion criteria. The most recent pilot study is presented in full in the supplementary material. All materials, data and an R script detailing the analyses are available online as part of the Open Science Framework by following this link - https://goo.gl/AiDpAS

\section{Materials and Apparatus}

The experiment was conducted in a dark, soundproof room and administered with E-Prime 2.0 Professional (SP2.0) on a 19” LED computer monitor (Resolution: 1900x1200; Refresh rate: $60 \mathrm{~Hz}$ ). Responses were collected with a standard computer keyboard. Participants wore over-ear headphones and placed their left and right index fingers over the ' $\mathrm{W}$ ' and ' $\mathrm{O}$ ' keys for the duration of the experiment. The stimulus set consisted, first, of two auditory stimuli: a $200 \mathrm{~ms} 440 \mathrm{~Hz}$ tone, which served as a metronome beat, as well as a $200 \mathrm{~ms}$ 'chirp', a smooth tone which ascended from $200 \mathrm{~Hz}$ to $800 \mathrm{~Hz}$ over $200 \mathrm{~ms}$. Visual stimuli consisted of two images of egocentric left and right hands with artificial 'shadows', as if viewed from above (each with visual angle of approximately $16^{\circ}$ vertically and $14^{\circ}$ horizontally). The hands were first presented with the fingers in a neutral position, and then with the left or right index finger depressed, giving an impression of apparent motion (a downward finger press).

\section{Procedure}

The experiment consisted of 4 blocks of 24 trials. In each trial, participants were first instructed about the particular rhythm they had to imagine. An image of the two left and right 


\section{INTENTION INSERTION}

hands was presented, from an egocentric perspective, and participants heard a slow, steady metronome beat $(440 \mathrm{~Hz}$ tone presented at $800 \mathrm{~ms}$ interval). An arrow moved, in time with the metronome beat, between the left or right index fingers, indicating a simple four-beat finger tapping sequence (Fig. 1). For any given trial, participants could encounter one of 6 different finger tapping patterns which consisted of all possible permutations of a 4-beat sequence involving two left and two right index finger movements. Participants were asked to imagine tapping along with this sequence and memorize it in this manner.

After four beats the arrow disappeared, but the metronome continued playing. Participants were instructed to continue to mentally rehearse the finger tapping sequence in time with the metronome, while refraining from overt movement. The hands remained onscreen in neutral position along with a central fixation cross that flashed in time with the metronome beat, providing an audio-visual representation of tempo, and drawing participants' attention to a point between the onscreen hands. After a variable duration of 5-8 beats, the word "Go!" appeared for $2000 \mathrm{~ms}$ and participants were asked to perform the keypress they happened to be imagining at that point (allowing us to measure task compliance). Participants were explicitly instructed to refrain from overt movement before this go signal. It was emphasized to even refrain from moving when surprising visual stimuli would appear during the imagination period.

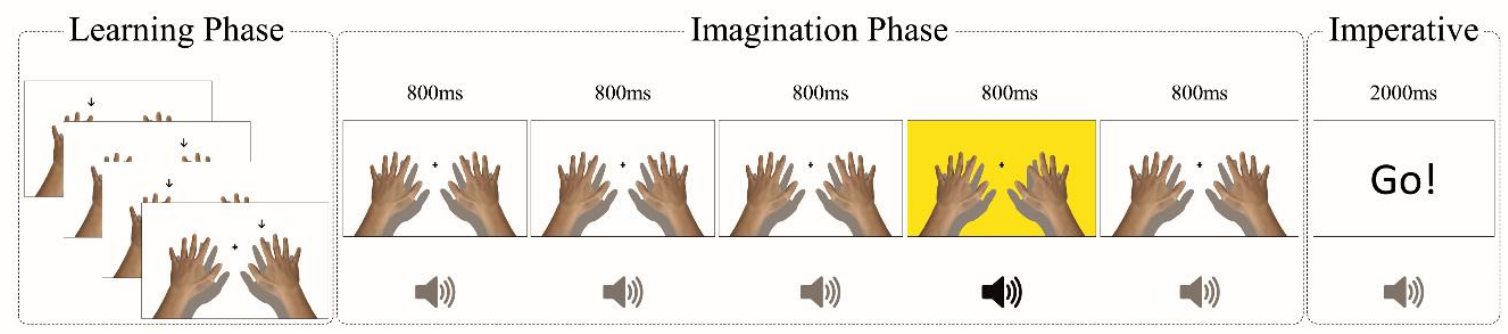

Figure 1. A schematic representation of a compatible cued trial beats from the Test Phase in our experiment. The depicted sequence is Left - Right - Left - Right - indicated by the arrows shown in the Learning Phase. In the subsequent five beat Imagination Phase, a Right index finger cue (screen colour, deviant tone and observed 


\section{INTENTION INSERTION}

finger movement) falls on beat four. The cue is presented when the participant is imagining a Right response and so this is compatible with the concurrent imagined response. We recorded the presence or absence of an action slip (keypress) in the $800 \mathrm{~ms}$ inter-beat period following cue onset. Participants were told to focus on their imagery and refrain from overt movement until presentation of the probe stimulus, "Go!"

The crucial manipulation was that in 8 of the 24 trials in each block, unexpected action effect cues appeared at the onset of one of the metronome beats (on either beat, 6,7 or 8 ). The action effect cues consisted of images of the left or right index fingers moving downwards and back to a neutral position, mirroring what participants would see if they actually carried out a finger tap themselves. Action effects were presented in tandem with a background colour change to yellow to increase the salience of the events. The finger movement cues were either compatible or incompatible with the finger movement that participants were currently imagining (if the participant correctly followed the tapping sequence). Within these 32 cued trials, all combinations of cue compatibility (compatible/incompatible), cued finger (left/right), trial length (5, 6, 7 and 8 beats) and cue placement in the sequence ( 1 or 2 beats prior to sequence end) occurred once and were therefore fully counterbalanced.

Prior to the main task, participants completed a short practice of 8 trials in which feedback was provided and incorrect responses were repeated. Participants were given the option of repeating the practice if required. The experimenter monitored participants' performance in this practice session. He emphasized that participants' ability to imagine - not execute - the movements was being tested. He instructed them not to move their fingers in the imagery interval and intervened if this instruction was not followed. He also unobtrusively remained in the room during the experiment proper in order to intervene if active finger movements were detected.

\section{Results}




\section{INTENTION INSERTION}

The procedure and analysis strategy was first developed in a pilot experiment $(N=26$; reported in full in the supplementary material) and then replicated here. This pilot study only differed from the current experiment in that the tones that accompanied the visual cues were lateralised (i.e. a left-panned tone for depressed left fingers, right-panned tone for depressed right fingers), but was otherwise identical, including all analysis steps and exclusion criteria. Please see the end of the Results section for the main results of this pilot study.

We used the percentage of correct responses to our probe stimulus (i.e. when "Go!" appeared and participants executed the currently imagined action) during trials which did not contain visual action cues to assess whether participants were engaging in the imagery task. Because all further analyses depend on task compliance, and as in the pilot experiment, we set conservative criteria and only considered participants with accuracy rates within one standard deviation of the group mean (chance $=50 \% ; M=84.3 \% ; S D=15.9 \%$ ) leading to the removal of 4 participants (leaving $N=28$ ). Exclusion of these participants does not affect the results. All results remain significant even when these participants are included.

First, to check whether participants followed our instruction to refrain from actively moving their fingers in the imagery intervals, we compared the number of slips on non-cued trials. In the absence of visual stimuli, accidental button presses were exceedingly rare and occurred on only $0.1 \%$ of beat intervals. These non-cued slips were roughly equally distributed between button presses with the imagined and non-imagined finger, suggesting that participants were not using finger movements to rehearse the imagined pattern (14 imagined vs. 10 non-imagined slips across all participants, $\left.\chi^{2}=.667, p=.414\right)$.

The data were analysed using generalised linear mixed-effects regression, GLMER, using the glmer() function of the lme4 package (Bates, Mächler, Bolker, \& Walker, 2015) in R Version 


\section{INTENTION INSERTION}

3.4.2 (R Core team, 2017). When modelling binary responses with GLMER, null hypothesis significance testing is implemented by comparing a full model and a reduced model without the fixed effect of interest with a likelihood ratio test (hereafter, LRT). The resulting chisquare test statistic is analogous to the F-statistic in conventional models. We report the estimate given by the GLMER model along with a bootstrapped $95 \%$ confidence interval based on 1000 simulations. All categorical predictors are effect coded and when referring to model parameters we capitalise the first letter for ease of comprehension.

Analysis proceeded in three steps. We first tested whether the surprising visual stimuli would induce unintended key presses (i.e. action slips) in the imagination period. To do this, each $800 \mathrm{~ms}$ inter-beat period (hereafter, simply 'Beat') was treated as a discrete event in which an action slip could have occurred or not (coded one or zero respectively). The model included fixed effects of Cue Presence (2 levels: Yes/No) and Trial (1-96, logged and mean centred) and a random intercept for each Participant and each Beat. In addition, we allowed the effect of Cue Presence, Trial and their interaction to vary within Participant by including random slopes for these terms. Such an approach is roughly analogous to a repeated measures oneway ANOVA with the factor Cue Presence. As predicted, the presence of unexpected cues significantly increased the probability of action slips, LRT: $\chi^{2}(1)=56.94, p<.001$; GLMER estimate: $\beta=4.2,95 \% \mathrm{CI}=[3.55,5.26]$. Predicted probabilities derived from repeated simulation of our model reveal that there was a $8.57 \%[3.89,18.03]$ chance of an action slip following the onset of a cue, but only .09\% $[.03, .22]$ when no cue was present. In addition, Cue Presence and Trial interacted, LRT: $\chi^{2}(1)=8.63, p=.003$; GLMER estimate: $\beta=-.79$, $95 \% \mathrm{CI}=[-1.46,-.34]$, indicating that cues became less effective at eliciting action slips over the course of the experiment. We did not find evidence of a main effect of Trial on the probability of an action slip occurring. 


\section{INTENTION INSERTION}

The second step of the analysis tested whether general action slips (i.e. any keypress response) would primarily be elicited by cues that match the currently imagined finger key press, not by those that mismatched. We constructed a smaller dataset only consisting of intervals in which cues were present (i.e. each participant contributed 32 observations), coded for whether the cue was compatible or incompatible with the currently imagined action. The model included fixed effects of Perception-Imagery Compatibility (2 levels: Compatible/Incompatible) and Cue Position within the trial series (ranked $1^{\text {st }}$ to $32^{\text {nd }}, \log$ transformed and mean centred) and a random intercept for each Participant. In addition, we added random slopes for Perception-Imagery Compatibility, Cue Position and their interaction, which allowed each participant to vary in their general response bias. The analysis revealed a significant main effect of Cue Position, LRT: $\chi^{2}(1)=44.43, p<.001$; GLMER estimate: $\beta=-1.36,95 \% \mathrm{CI}=[-1.79,-1.12]$, reflecting the fact that action slips were most likely in the early stages of the experiment and became less frequent as it progressed. Importantly, as predicted, there was a main effect of Perception-Imagery Compatibility, LRT: $\chi^{2}(1)=7.59, p=.006$; GLMER estimate: $\beta=.54,95 \% \mathrm{CI}=[.22,1.07]$. Action slips were more likely when observed finger press and the imagined finger press were identical compared to when they were different. In addition, Perception-Imagery Compatibility and Cue Position interacted, LRT: $\chi^{2}(1)=5.59, p=.018$; GLMER estimate: $\beta=.34,95 \% \mathrm{CI}=$ $[.07, .76]$. Figure 2 , middle panel, suggests that this is because action slip frequency was roughly equivalent in the compatible and incompatible conditions early in the session (when the number of action slips across both conditions was high), but that the difference between the compatible and incompatible conditions emerged later in the session when participants had adjusted to the surprising cues and the total number of slips had declined. 
(a)

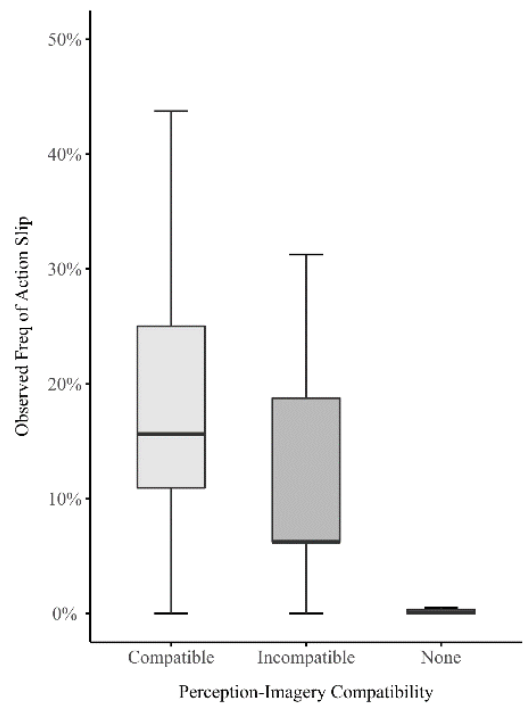

(b)

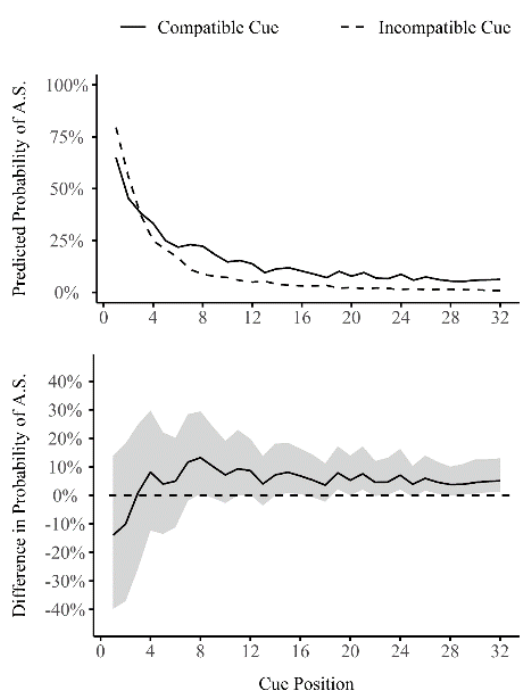

(c)

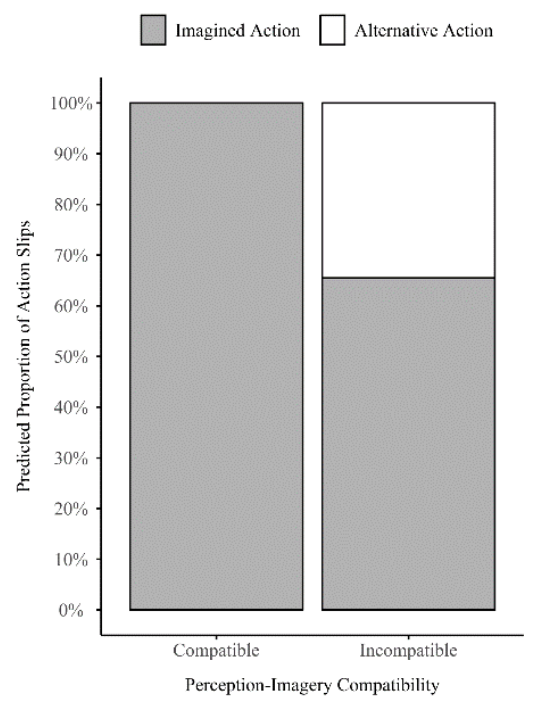

Figure 2. Observed frequencies and predicted probabilities of general and specific action slips by condition. Four plots showing, from left to right; (a) a box plot of the observed frequency of action slips produced by each participant, broken down by Perception-Imagery Compatibility with a thick black bar to represent the median, a box showing the interquartile range and whiskers to indicate maxima and minima, (b) a combination plot indicating the predicted probability of action slips across the experiment according to Perception-Imagery Compatibility and the associated difference plot (i.e. Compatible - Incompatible) in which the shaded area shows a 95\% confidence interval derived by simulation, and (c) a stacked bar plot indicating the predicted proportion of specific action slips (i.e. either the imagined action or alternative action) made according to Perception-Imagery Compatibility, derived by simulation.

Finally, we tested the hypothesis that specific action slips (i.e. the specific keypress response) should generally reflect the currently imagined key press, and that the alternative action would primarily be elicited by an incompatible cue. We created a new dataset consisting of only observations in which an action slip occurred and added a new outcome variable indicating whether the participant had executed the imagined or alternative action (coded one and zero respectively). The model included fixed effects of Perception-Imagery

Compatibility, Cue Position and their interaction along with a random intercept for each Participant. In addition, we included a random slope for Perception-Imagery Compatibility. The test revealed a significant main effect of Perception-Imagery Compatibility, LRT: $\chi 2$ (1) $=19.01, p<.001$; GLMER estimate: $\beta=5.73,95 \% \mathrm{CI}=[3.81,16.19]$. Predicted probabilities, derived by simulation, reveal that there was a $100 \%[93.83,100]$ chance that participants would execute the imagined response when faced with a compatible cue, but only a $65.57 \%[49,79.2]$ chance in response to an incompatible cue (Figure 2, right panel). 


\section{INTENTION INSERTION}

Importantly, confidence intervals for compatible cues in particular did not overlap with chance (i.e. 50\%), showing that participants were much more likely to produce the currently imagined response compared to the alternative response. Cue Position and the interaction between Perception-Imagery Compatibility and Cue Position did not affect which action was executed.

These results replicate our pilot study (reported in full in the supplementary material). Specifically, the pilot study showed that action slips were more likely following visual cues relative to when no cue was present $\left(\right.$ LRT: $\chi^{2}(1)=30.1, p<.001$; GLMER estimate: $\beta=$ $3.15,95 \% \mathrm{CI}=[2.28,3.87])$, that action slips were more likely following compatible rather than incompatible visual cues (LRT: $\chi^{2}(1)=4.91, p=.027$; GLMER estimate: $\beta=-.83,95 \%$ $\mathrm{CI}=[.21,3.99])$, and that participants were more likely to press with the non-imagined finger when presented with an incompatible cue that primed the non-imagined response, relative to a compatible cue $\left(\mathrm{LRT}: \chi^{2}(1)=9.52, p=.002\right.$; GLMER estimate: $\beta=2.64,95 \% \mathrm{CI}=[1.62$, 22.2]).

\section{General Discussion}

We asked participants to imagine - but not execute - sequences of finger movements, and unexpectedly presented them, at some point during their imagination, with visual images of these actions being executed. If forming an action intention is equivalent to activating the relevant action effect codes, then the summed activation of these codes through imagery and perception may suffice to make them super-threshold, causing participants to sometimes execute even movements that they were asked to withhold. Such effects would go beyond prior work showing that observing (or imagining) actions modulates selection or executing planned actions - speeding up responses to congruent cues or inducing subtle changes in 


\section{INTENTION INSERTION}

associated muscle activity - and confirm a long-held but untested assumption of ideomotor models: that activating an action's effects is equivalent to inducing a motor intention that leads to overt action when activated strongly enough.

Consistent with these predictions, the combination of imagined and observed action effects effectively induced non-willed button presses. This effect was strongest when the observed and imagined actions were identical, and was weaker when they were different. This rules out the possibility that the action slips were merely startle responses or the release of prepared actions triggered by surprising stimuli (Brown et al., 1991; Maslovat et al., 2013) because this should occur equally for compatible and incompatible stimuli. Instead, it is consistent with the hypothesized summation of action effect codes, which can happen only if imagined and observed action effects are compatible, but not if they are incompatible. The data therefore show for the first time that activating an action's perceptual consequences does not only support action planning or selection (see Keller \& Koch, 2006, for an application to similar finger sequences as used here), but - in line with ideomotor models - is equivalent to forming an action intention, which when strong enough, suffices to trigger action.

Two further aspects of our results confirm this interpretation. First, the effect of cue-imagery summation emerged later in the experiment and led to a persistent increase in action slips when visual and imagined action were identical, again ruling out that action slips reflect mere startle responses or a release of pre-planned responses. Second, presentation of action effects primarily caused participants to execute the currently imagined action, compared to the alternative finger press. As predicted, the frequency with which the alternative (nonimagined) action was executed only increased when imagined and presented action effects were not identical, and the visual action cue therefore activated the currently non-imagined action (replicating the results of the pilot experiment). This shows that two simultaneously 


\section{INTENTION INSERTION}

activated actions intentions - one derived from imagery and one from observation - compete with each other, such that both can trigger involuntary responses independently, albeit less frequently than if both plans are identical and can summate (Hommel, 2009; Hommel et al., 2001).

Previous work has characterised action initiation as an integration-to-bounds mechanism, which accumulates evidence for appropriate actions and executes the most suitable one if a threshold is reached, irrespective of whether this evidence results from the evaluation of imperative task cues or from internal decisions about whether to act or to wait (Churchland et al., 2008; Murakami et al., 2014). Once an action has surpassed the threshold, it triggers an "avalanche" of motor processes, supported by deep brain structures such as the basal ganglia, which facilitate fluent execution. Our data is entirely consistent with this view but reveals, for the first time, that activated action effects - either through imagery or action observation can stand in for this accumulation of evidence, making the decision super-threshold without additional processing. The data therefore provide direct evidence for the notion that forming an action intention may be identical to activating - imagining - the action's effects, which is executed when its activation is strong enough (Prinz, 1997; Stock \& Stock, 2004). Our findings go beyond prior research that has shown only that motor imagery (Ramsey et al., 2010), action observation (e.g. Bach, Bayliss, \& Tipper, 2011; Bach et al., 2007; Brass et al., 2001) and learned action effect associations (e.g. Elsner \& Hommel, 2001; Kunde et al., 2004) can modulate the selection, speed and accuracy of already planned responses, but which has left open whether action effect activation suffices to elicit actions independently.

An open question is how an action-effect based control of action is implemented. We have assumed that activated action effects induce action intentions by adding activation to one particular action until it becomes super-threshold, so that it is executed. However, it is also 


\section{INTENTION INSERTION}

possible that either the visual or the imagery-based action effects act by removing inhibition from one particular action (e.g. Rieger, Dahm, \& Koch, 2017), in line with James' dictum (1890) that a mental idea of an action will lead to overt execution, as long as it is "not kept from so doing by an antagonistic representation present simultaneously to the mind" (p. 526). We are agnostic about these two possibilities. On a conceptual level, both are equivalent, as both summation of activation and removal of inhibition have a net positive effect in bringing activation closer to threshold and inducing overt behaviour. Indeed, intentions to not act have been shown to be similarly controlled by mental images of non-action (e.g. Kühn, Elsner, Prinz, \& Brass, 2009; Ridderinkhof, van den Wildenberg, \& Brass, 2014), and both inhibiting and excitatory roles have been attributed to complementary basal ganglia-prefrontal circuits for action execution (for a recent review, see Calabresi, Picconi, Tozzi, Ghiglieri, \& Di Filippo, 2014). However, while our study was not designed to resolve this issue, we believe that it may provide tentative support for an "energizing" role of action effect activation. If the visual action cues would primarily act through removing inhibition, then they should have induced action slips only for the currently imagined action; removing inhibition should not cause a fully-fledged button press of the un-imagined finger. This was not the case. Even the currently non-imagined action was executed - albeit less frequently than the imagined response - if it was so cued by the respective visual action cue, providing some evidence for an additive, "energizing" influence on action effect codes.

Action in the real world has been conceptualised as an interaction of higher-level goals with lower-level cues from the environment: actions are executed if they match both the individual's goals and are afforded by the environment (Cisek, 2007; Guérard \& Brodeur, 2015; Kunde, Elsner, \& Kiesel, 2007; Ondobaka \& Bekkering, 2012). Our experimental paradigm can be considered as a model for these interactions. In ideomotor frameworks, higher-level goals are nothing else than the imagined action effects one wants to achieve 


\section{INTENTION INSERTION}

(Hommel et al., 2001), similar to the imagined actions in the present study. Our study shows that bottom-up activity induced by perceptual cues in the environment - in our case the observed actions - activate the same effect codes, and that we execute actions that are consistent with both. It may therefore explain why many everyday actions are executed seemingly automatically, simply because they match the goals one has previously committed to and the structure of the environment (Bargh \& Chartrand, 1999; Kunde et al., 2007), or why one's longer-term action plans (e.g. healthy eating) are followed more easily when one has visualised one's behaviour in the relevant situation (Brandstatter, Lengfelder, \& Gollwitzer, 2001). They may also explain the wide range of action slips that people make, where they sometimes perform unintended actions (e.g., putting their car keys in the fridge while leaving milk out on the table or making a wrong, but habitual, turn at a traffic junction), find themselves copying the postures and mannerisms of others (Chartrand \& Bargh, 1999), or respond, in a seemingly non-intentional way, to ideomotor suggestions during hypnosis (e.g. an arm levitation suggestion).

\section{Conclusion}

How do people "will" their actions? We found that activation of action effects through imagery and perception was sufficient to elicit actions of the participants, even when instructed to be on guard against such slips. This provides direct evidence for the central tenet of ideomotor models, that forming an action intention is equivalent to bringing to mind the action's perceptual effects, and that a motor intention is nothing else than the activated action effects crossing a response threshold. Our results link voluntary control of action in everyday life to classical ideomotor effects in hypnosis and related phenomena (Chevreul's pendulum, Ouija board), providing direct support for James' (1890) claim that ideomotor control of 


\section{INTENTION INSERTION}

action is not "a curiosity" but the "normal process" through which people control their own actions.

\section{Context}

This research was motivated by classical ideomotor phenomena (i.e. Chevreul's Pendulum) wherein people produce movements that they report feel "unintentional". We are interested in how such behaviour could arise in the absence of a specific intention and, more specifically, whether we can effectively insert an intention to act by manipulating the interacting processes of action, perception and imagery. This experiment is one part of an ongoing programme of research by the first author and his supervisory team into the role of action effect representations in the chain of events which links goals, intentions, actions and their sensory consequences. The supervisory team have published widely on topics related to perceptual anticipation, including ideomotor action control (Bach et al., 2010), predictive social perception (Bach, Nicholson, \& Hudson, 2014; Bach \& Schenke, 2017; Hudson, Nicholson, Simpson, Ellis, \& Bach, 2016) and the role of associative learning in environmentally-cued actions (Hardy, Mitchell, Seabrooke, \& Hogarth, 2017; Seabrooke, Le Pelley, Hogarth, \& Mitchell, 2017). 


\section{INTENTION INSERTION}

\section{References}

Adams, R. A., Shipp, S., \& Friston, K. J. (2013). Predictions not commands: Active inference in the motor system. Brain Structure and Function, 218(3), 611-643. https://doi.org/10.1007/s00429-012-0475-5

Bach, P., Allami, B. K., Tucker, M., \& Ellis, R. (2014). Planning-related motor processes underlie mental practice and imitation learning. Journal of Experimental Psychology: General, 143(3), 1277-1294. https://doi.org/10.1037/a0035604

Bach, P., Bayliss, A., \& Tipper, S. (2011). The predictive mirror: interactions of mirror and affordance processes during action observation. Psychonomic Bulletin \& Review, 18(1), 171-176. https://doi.org/10.3758/s13423-010-0029-x

Bach, P., Griffiths, D., Weigelt, M., \& Tipper, S. P. (2010). Gesturing Meaning: Non-action Words Activate the Motor System. Frontiers in Human Neuroscience, 4(November), 214. https://doi.org/10.3389/fnhum.2010.00214

Bach, P., Nicholson, T., \& Hudson, M. (2014). The affordance-matching hypothesis: how objects guide action understanding and prediction. Frontiers in Human Neuroscience, 8 , 254. https://doi.org/10.3389/fnhum.2014.00254

Bach, P., Peatfield, N. A., \& Tipper, S. P. (2007). Focusing on body sites: The role of spatial attention in action perception. Experimental Brain Research, 178(4), 509-517. https://doi.org/10.1007/s00221-006-0756-4

Bach, P., \& Schenke, K. C. (2017). Predictive social perception: Towards a unifying framework from action observation to person knowledge. Social and Personality 


\section{INTENTION INSERTION}

Psychology Compass, 11(7). https://doi.org/10.1111/spc3.12312

Badets, A., Koch, I., \& Philipp, A. M. (2016). A review of ideomotor approaches to perception, cognition, action, and language: advancing a cultural recycling hypothesis. Psychological Research, 80(1), 1-15. https://doi.org/10.1007/s00426-014-0643-8

Bargh, J. A., \& Chartrand, T. L. (1999). The Unbearable Automaticity of Being. American Psychologist, 54(7), 462-479. https://doi.org/10.1037/0003-066X.54.7.462

Bates, D., Mächler, M., Bolker, B., \& Walker, S. (2015). Fitting Linear Mixed-Effects Models Using lme4. Journal of Statistical Software, 67(1), 1-48. https://doi.org/10.18637/jss.v067.i01

Berends, H. I., Wolkorte, R., Ijzerman, M. J., \& Van Putten, M. J. A. M. (2013). Differential cortical activation during observation and observation-and-imagination. Experimental Brain Research, 229(3), 337-345. https://doi.org/10.1007/s00221-013-3571-8

Brandstatter, V., Lengfelder, A., \& Gollwitzer, P. M. (2001). Implementation Intentions and Efficient Action Initiation. Journal of Personality and Social Psychology, 81(5), 946960. https://doi.org/10.1037//0022-35I4.81.5.946

Brass, M., Bekkering, H., \& Prinz, W. (2001). Movement observation affects movement execution in a simple response task. Acta Psychologica, 106(1-2), 3-22. https://doi.org/10.1016/S0001-6918(00)00024-X

Brown, P., Rothwell, J. C., Thompson, P. D., Britton, T. C., Day, B. L., \& Marsden, C. D. (1991). New observations on the normal auditory startle reflex in man. Brain, 114(4), 1891-1902. https://doi.org/10.1093/brain/114.4.1891 


\section{INTENTION INSERTION}

Calabresi, P., Picconi, B., Tozzi, A., Ghiglieri, V., \& Di Filippo, M. (2014). Direct and indirect pathways of basal ganglia: a critical reappraisal. Nature Neuroscience, 17(8), 1022.

Chartrand, T. L., \& Bargh, J. a. (1999). The Chameleon Effect: The Perception-Behaviour Link and Social Interaction. Journal of Personality and Social Psychology, 76(6), 893910. https://doi.org/10.1037/0022-3514.76.6.893

Chevreul, M. E. (1833). Lettre à M. Ampère sure une classe particulaires. Review Des Deux Mondes, (2), 258-266.

Churchland, A. K., Kiani, R., \& Shadlen, M. N. (2008). Decision-making with multiple alternatives. Nature Neuroscience, 11(6), 693-702. https://doi.org/10.1038/nn0708-851c

Cisek, P. (2007). Cortical mechanisms of action selection: the affordance competition hypothesis. Philosophical Transactions of the Royal Society of London. Series B, Biological Sciences, 362(1485), 1585-1599. https://doi.org/10.1098/rstb.2007.2054

Easton, R. D., \& Shor, R. E. (1975). Information processing analysis of the Chevreul pendulum illusion. Journal of Experimental Psychology: Human Perception and Performance, 1(3), 231-236. https://doi.org/10.1037/0096-1523.1.3.231

Easton, R. D., \& Shor, R. E. (1976). An Experimental Analysis of the Chevreul Pendulum Illusion. The Journal of General Psychology, 95(1), 111-125. https://doi.org/10.1080/00221309.1976.9710871

Easton, R. D., \& Shor, R. E. (1977). Augmented and Delayed Feedback in the Chevreul Pendulum Illusion. The Journal of General Psychology, 97(2), 167-177. 


\section{INTENTION INSERTION}

https://doi.org/10.1080/00221309.1977.9920835

Elsner, B., \& Hommel, B. (2001). Effect anticipation and action control. Journal of Experimental Psychology. Human Perception and Performance, 27(1), 229-240. https://doi.org/10.1037/0096-1523.27.1.229

Friston, K. (2011). What is optimal about motor control? Neuron, 72(3), 488-498. https://doi.org/10.1016/j.neuron.2011.10.018

Guérard, K., \& Brodeur, M. (2015). Manipulability agreement as a predictor of action initiation latency. Behavior Research Methods, 47(2), 572-579. https://doi.org/10.3758/s13428-014-0495-6

Hardy, L., Mitchell, C., Seabrooke, T., \& Hogarth, L. (2017). Drug cue reactivity involves hierarchical instrumental learning: evidence from a biconditional Pavlovian to instrumental transfer task. Psychopharmacology, 234(13), 1977-1984. https://doi.org/10.1007/s00213-017-4605-X

Hommel, B. (1995). Stimulus-response compatibility and the Simon effect: Toward an empirical clarification. Journal of Experimental Psychology: Human Perception and Performance, 21(4), 764-775. https://doi.org/10.1037/0096-1523.21.4.764

Hommel, B. (2009). Action control according to TEC (theory of event coding). Psychological Research, 73(4), 512-526. https://doi.org/10.1007/s00426-009-0234-2

Hommel, B., Müsseler, J., Aschersleben, G., \& Prinz, W. (2001). The Theory of Event Coding (TEC): A framework for perception and action planning. Behavioral and Brain Sciences, 24(5), 849-878. https://doi.org/10.1017/S0140525X01000103 


\section{INTENTION INSERTION}

Hudson, M., Nicholson, T., Simpson, W. A., Ellis, R., \& Bach, P. (2016). One Step Ahead: The Perceived Kinematics of Others' Actions Are Biased Toward Expected Goals. Journal of Experimental Psychology: General, 145(1), 1-7. https://doi.org/10.1037/xge0000126

James, W. (1890). The principles of psychology (Vols. 1 \& 2). New York, NY: Henry Holt and Company. https://doi.org/10.1037/10538-000

Keller, P. E., \& Koch, I. (2006). The planning and execution of short auditory sequences. Psychonomic Bulletin \& Review, 13(4), 711-716. https://doi.org/10.3758/BF03193985

Kühn, S., Elsner, B., Prinz, W., \& Brass, M. (2009). Busy doing nothing: Evidence for nonaction-effect binding. Psychonomic Bulletin \& Review, 16(3), 542-549. https://doi.org/10.3758/PBR.16.3.542

Kühn, S., Keizer, A., Rombouts, S. A. R. B., \& Hommel, B. (2011). The functional and neural mechanism of action preparation: roles of EBA and FFA in voluntary action control. Journal of Cognitive Neuroscience, 23(1), 214. https://doi.org/10.1162/jocn.2010.21418

Kunde, W., Elsner, K., \& Kiesel, A. (2007). No anticipation-no action: The role of anticipation in action and perception. Cognitive Processing, 8(2), 71-78. https://doi.org/10.1007/s10339-007-0162-2

Kunde, W., Koch, I., \& Hoffmann, J. (2004). Anticipated action effects affect the selection, initiation, and execution of actions. Quarterly Journal of Experimental Psychology: Part A: Human Experimental Psychology, 57(1), 87. 


\section{INTENTION INSERTION}

https://doi.org/10.1080/02724980343000143

Maslovat, D., Chua, R., \& Hodges, N. J. (2013). When unintended movements "leak" out: A startling acoustic stimulus can elicit a prepared response during motor imagery and action observation. Neuropsychologia, 51(5), 838-844. https://doi.org/10.1016/j.neuropsychologia.2013.01.016

Murakami, M., Vicente, M. I., Costa, G. M., \& Mainen, Z. F. (2014). Neural antecedents of self-initiated actions in secondary motor cortex. Nature Neuroscience, 17(11), 1574-82. https://doi.org/10.1038/nn.3826

Ondobaka, S., \& Bekkering, H. (2012). Hierarchy of idea-guided action and perceptionguided movement. Frontiers in Psychology, 3(December), 1-5. https://doi.org/10.3389/fpsyg.2012.00579

Pacherie, E., \& Haggard, P. (2010). What are intentions? In W. Sinnott-Armstrong \& N. Lynn (Eds.), Conscious Will and Responsibility. A tribute to Benjamin Libet (pp. 7084). Oxford University Press, USA.

Prinz, W. (1997). Perception and Action Planning. European Journal of Cognitive Psychology, 9(2), 129-154. https://doi.org/10.1080/713752551

R Core team. (2017). R: A Language and Environment for Statistical Computing. Vienna, Austria.: R Foundation for Statistical Computing. Retrieved from https://www.rproject.org/

Ramsey, R., Cumming, J., Eastough, D., \& Edwards, M. G. (2010). Incongruent imagery interferes with action initiation. Brain and Cognition, 74(3), 249-254. 


\section{INTENTION INSERTION}

https://doi.org/10.1016/j.bandc.2010.08.005

Ridderinkhof, K. R., van den Wildenberg, W. P. M., \& Brass, M. (2014). “Don't” versus "Won't": Principles, mechanisms, and intention in action inhibition. Neuropsychologia, 65(September), 255-262. https://doi.org/10.1016/j.neuropsychologia.2014.09.005

Rieger, M., Dahm, S., \& Koch, I. (2017). Inhibition in motor imagery: a novel action mode switching paradigm. Psychonomic Bulletin \&amp; Review, 24(2), 459-466. https://doi.org/10.3758/s13423-016-1095-5

Schurger, A., Mylopoulos, M., \& Rosenthal, D. (2016). Neural Antecedents of Spontaneous Voluntary Movement: A New Perspective. Trends in Cognitive Sciences, 20(2), 77-79. https://doi.org/10.1016/j.tics.2015.11.003

Seabrooke, T., Le Pelley, M. E., Hogarth, L., \& Mitchell, C. J. (2017). Evidence of a GoalDirected Process in Human Pavlovian-Instrumental Transfer. Journal of Experimental Psychology: Animal Learning and Cognition, 43(4), 377-387. https://doi.org/10.1037/xan0000147

Shin, Y. K., Proctor, R. W., \& Capaldi, E. J. (2010). A review of contemporary ideomotor theory. Psychological Bulletin, 136(6), 943-974. https://doi.org/10.1037/a0020541

Stock, A., \& Stock, C. (2004). A short history of ideo-motor action. Psychological Research, 68(2-3), 176-188. https://doi.org/10.1007/s00426-003-0154-5

Tecuapetla, F., Jin, X., Lima, S. Q., \& Costa, R. M. (2016). Complementary Contributions of Striatal Projection Pathways to Action Initiation and Execution. Cell, 166(3), 703-715. https://doi.org/10.1016/j.cell.2016.06.032 


\section{INTENTION INSERTION}

Tucker, M., \& Ellis, R. (1998). On the relations between seen objects and components of potential actions. Journal of Experimental Psychology: Human Perception and Performance, 24(3), 830-846. https://doi.org/10.1037/0096-1523.24.3.830

Turella, L., Tucciarelli, R., Oosterhof, N. N., Weisz, N., Rumiati, R., \& Lingnau, A. (2016). Beta band modulations underlie action representations for movement planning. NeuroImage, 136, 197-207. https://doi.org/10.1016/j.neuroimage.2016.05.027

van Steenbergen, H., Warren, C. M., Kühn, S., de Wit, S., Wiers, R. W., \& Hommel, B. (2017). Representational precision in visual cortex reveals outcome encoding and reward modulation during action preparation. NeuroImage, 157, 415-428. https://doi.org/10.1016/j.neuroimage.2017.06.012

Vargas, C. D., Olivier, E., Craighero, L., Fadiga, L., Duhamel, J. R., \& Sirigu, A. (2004). The Influence of Hand Posture on Corticospinal Excitability during Motor Imagery: A Transcranial Magnetic Stimulation Study. Cerebral Cortex, 14(11), 1200-1206. https://doi.org/10.1093/cercor/bhh080

Vogt, S., Di Rienzo, F., Collet, C., Collins, A., \& Guillot, A. (2013). Multiple roles of motor imagery during action observation. Frontiers in Human Neuroscience, 7(November), 807. https://doi.org/10.3389/fnhum.2013.00807

Waszak, F., Cardoso-Leite, P., \& Hughes, G. (2012). Action effect anticipation: Neurophysiological basis and functional consequences. Neuroscience and Biobehavioral Reviews, 36(2), 943-959. https://doi.org/10.1016/j.neubiorev.2011.11.004

Zimmermann, M., Mars, R., Lange, F., Toni, I., \& Verhagen, L. (2018). Is the extrastriate 


\section{INTENTION INSERTION}

body area part of the dorsal visuomotor stream? Brain Structure and Function, 223(1), 31-46. https://doi.org/10.1007/s00429-017-1469-0

Zimmermann, M., Verhagen, L., de Lange, F. P., \& Toni, I. (2016). The Extrastriate Body Area Computes Desired Goal States during Action Planning. eNeuro, 3(2). https://doi.org/10.1523/ENEURO.0020-16.2016

\section{Author Note}

This research was carried out as part of a $\mathrm{PhD}$ by J. A. Colton at Plymouth University under the supervision of Dr. P. Bach, Dr. B. Whalley and Prof. C. Mitchell (Director of Studies). The authors declare no competing financial interests.

\section{Contributions}

J. A. Colton developed the study concept. All authors contributed to the study design. Testing and data collection were performed by J. A. Colton. J. A. Colton performed the data analysis and interpretation under the supervision of B. Whalley. J. A. Colton drafted the manuscript, and all authors provided critical revisions. All authors approved the final version of the manuscript for submission.

\section{Acknowledgements}

This research was supported by a PhD studentship awarded to J. A. Colton by Plymouth University. This research was presented as a poster at the Rovereto Workshop on Concepts, Actions and Objects (CAOS, 2017), University of Trento, Italy, and as part of an oral presentation delivered at the Cognition Institute Conference (2016), Plymouth, UK. 


\section{INTENTION INSERTION}

\section{Figure Captions}

Figure 1. A schematic representation of a compatible cued trial beats from the Test Phase in our experiment. The depicted sequence is Left - Right - Left - Right - indicated by the arrows shown in the Learning Phase. In the subsequent five beat Imagination Phase, a Right index finger cue (screen colour, deviant tone and observed finger movement) falls on beat four. The cue is presented when the participant is imagining a Right response and so this is compatible with the concurrent imagined response. We recorded the presence or absence of an action slip (keypress) in the $800 \mathrm{~ms}$ inter-beat period following cue onset. Participants were told to focus on their imagery and refrain from overt movement until presentation of the probe stimulus, "Go!"

Figure 2. Observed frequencies and predicted probabilities of general and specific action slips by condition. Four plots showing, from left to right; (a) a box plot of the observed frequency of action slips produced by each participant, broken down by PerceptionImagery Compatibility with a thick black bar to represent the median, a box showing the interquartile range and whiskers to indicate maxima and minima, (b) a combination plot indicating the predicted probability of action slips across the experiment according to Perception-Imagery Compatibility and the associated difference plot (i.e. Compatible Incompatible) in which the shaded area shows a 95\% confidence interval derived by simulation, and (c) a stacked bar plot indicating the predicted proportion of specific action slips (i.e. either the imagined action or alternative action) made according to Perception Imagery Compatibility, derived by simulation. 


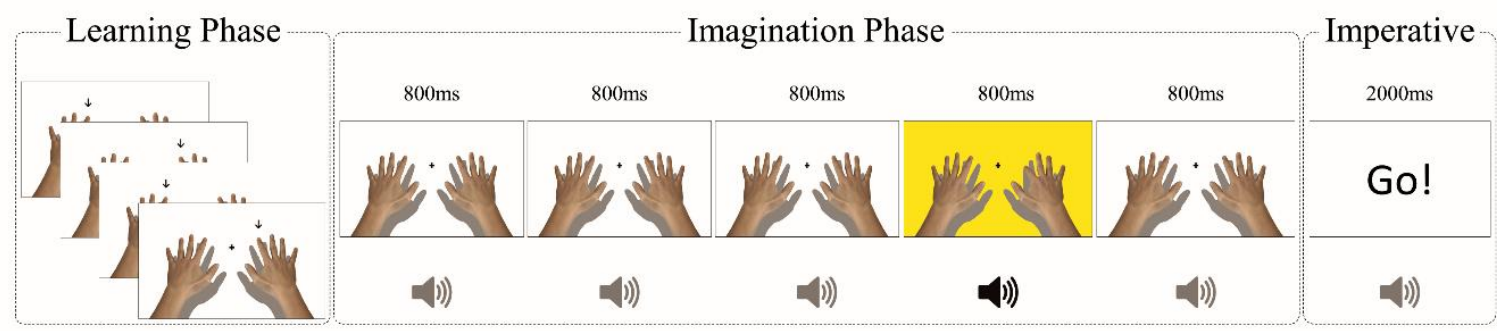




\section{INTENTION INSERTION}

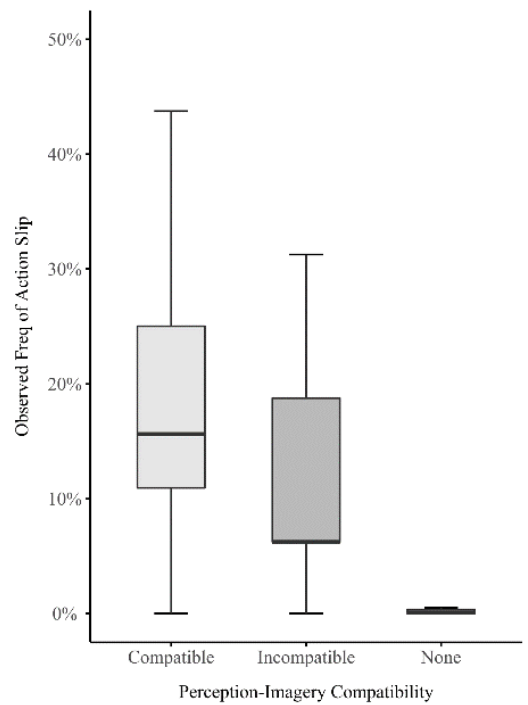

(b)

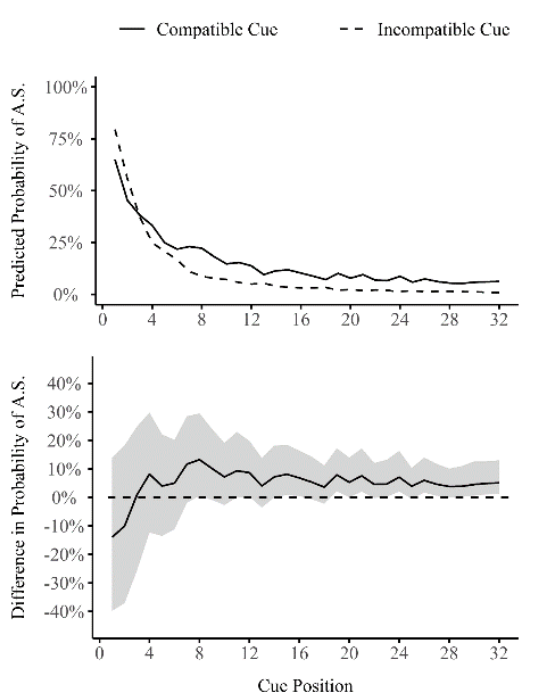

(c)

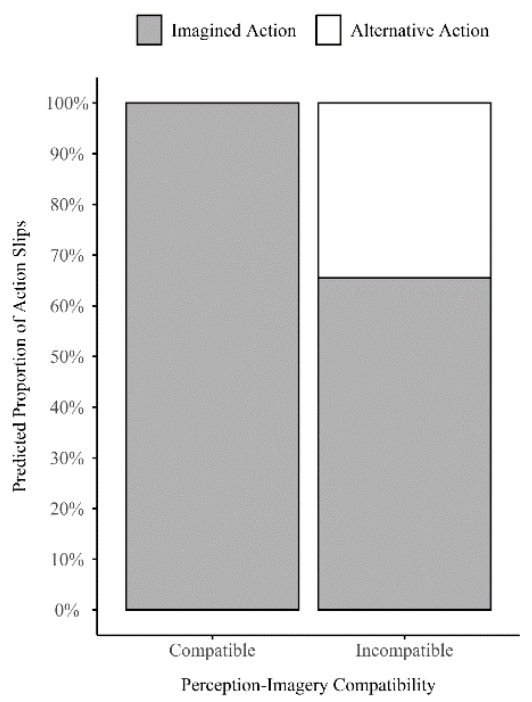

